\title{
Immunohistochemical Examinations of Sex Hormone Receptors in Benign Vocal Fold Lesions
}

\author{
Eleonore Cohen ${ }^{a} \quad$ Andrea Kolbus $^{b}$ Michael van Trotsenburg ${ }^{b}$ \\ Margarethe Rudas ${ }^{c}$ Reinhard Horvat ${ }^{c}$ Berit Schneider ${ }^{d}$ \\ ${ }^{a}$ Department of Obstetrics and Gynecology, St. Agnes Hospital, Bocholt, Germany; ${ }^{b}$ Department of Obstetrics and \\ Gynecology, Division of Gynecological Endocrinology and Reproductive Medicine, Medical University of Vienna, \\ 'Department of Pathology, Division of Gynecopathology and Senopathology and Cytology, University Hospital \\ of Vienna, and d Department of Otorhinolaryngology, Division of Phoniatrics-Logopedics, Medical University of \\ Vienna, Vienna, Austria
}

\section{Key Words}

Hormone receptors $\cdot$ Estrogen $\cdot$ Progesterone $\cdot$ Androgen receptor $\cdot$ Vocal folds $\cdot$ Immunohistochemistry $\cdot$ Vocal fold nodules $\cdot$ Reinke's edema $\cdot$ Vocal fold polyp

\begin{abstract}
Background: Acquired benign vocal fold lesions are among the most common causes of voice problems. Since the local impact of estrogen and progesterone receptors in laryngeal tissue is discussed controversially, the presence of sex hormone receptors in benign vocal fold alterations needs to be clarified. Goal of the Study: To investigate the expression of estrogen- $\alpha$ receptors (ER- $\alpha$ ), estrogen- $\beta$ receptors (ER- $\beta$ ), progesterone receptors (PR) and androgen receptors (AR) in acquired benign vocal fold alterations. Methods: Laryngeal epithelial specimens of 14 patients (13 female, 1 male) taken intraoperatively were investigated using immunohistochemistry in order to objectify ER- $\alpha, E R-\beta$, PR and AR. Macroscopically and histopathologically diagnosed edemas of Reinke's space ( $n=10)$, vocal fold polyps $(n=3)$ and vocal fold nodules $(n=1)$ were enrolled in this study. Results: No specific nuclear immunohistochemical staining could be seen in the biopsies taken. Only unspecific staining patterns could be observed. Conclusion: Sex hormone receptors
\end{abstract}

could not be detected in the specimens tested, thus, any direct influence of sex hormones on the development of benign vocal fold lesions is rather unlikely. The results of this study confirm the impact of vocal fold stress and biomechanical abnormalities on their development due to voice overstraining and abuse.

Copyright $\odot 2009$ S. Karger AG, Basel

\section{Introduction}

Acquired benign vocal fold lesions can be classified into separate entities by their location, gross appearance and vibration behavior: nodules, polyps and edemas. Vocal fold nodules are defined as small lesions occurring bilaterally on the border of the anterior and middle third of vocal folds. These lesions are confined to the epithelium and upper layer of the lamina propria. A vocal fold polyp might occur on the anterior third of the vocal fold, mostly on the free edge, sessile or pedunculate and very mobile. An edema of the free edge is to be seen as a uni-

Eleonore Cohen and Andrea Kolbus contributed equally to the work and should be regarded as joint first authors.

\section{KARGER}

Fax +41613061234 E-Mail karger@karger.ch www.karger.com
(C) 2009 S. Karger AG, Basel

$1021-7762 / 09 / 0615-0259 \$ 26.00 / 0$

Accessible online at:

www.karger.com/fpl
Berit Schneider, MD

Department of Otorhinolaryngology, Division of Phoniatrics-Logopedics

Medical University of Vienna, Währinger Gürtel 18-20

AT-1090 Vienna (Austria)

Tel. +43 140400 3310,Fax +43 140400 3332,E-Mail berit.schneider@meduniwien.ac.at 
lateral or bilateral bleaching-white transparent swelling of the vocal fold; due to the fluid in Reinke's space it is also termed Reinke's edema.

The etiology of these lesions encompasses multiple cofactors, not only behavioral or environmental, but also endogenous and inherited. It is widely believed that benign vocal fold lesions are the result of voice-related trauma due to vocal misuse and overuse since they occur more frequently in voice professionals [1-4]. Increased collision forces and increased Bernoulli separation forces are considered to be the underlying mechanism for these alterations based on their location and histological findings. Mechanical stress during vocal fold collision is assumed to be a main cause of benign vocal fold lesion formation based on observations of high velocity impact between vocal folds during phonation [5-7] and of structural disruption of the basement membrane of these lesions [8]. Computational models support and expand the hypothesis that benign vocal fold lesions are a reaction to mechanical stress $[2,8]$. As Gunter [8] pointed out, the magnitude of stress-induced components increases during collision in the region where pathologies often develop. Furthermore, Czerwonka et al. [9] have recently reported on vibration-induced rises in capillary pressure causing vocal nodules and edema.

While several lesions like vocal fold polyps can be seen in both men and women, especially vocal fold nodules seem to occur more often in female patients $[10,11]$. The relationship between voice and sex hormones in the female body is well established. Starting with subjective reports on voice alterations associated with hormonal changes, through acoustic voice sound analysis to identification of hormonal receptors in the larynx, it is evident that the larynx is affected by sex hormones and its fluctuations [12]. Several studies focused on premenstrual and menstrual, as well as postmenopausal changes. Abitbol et al. [13] found muscular and mucosal alterations including an increased number of vocal cord nodules in 59 of 97 women with premenstrual vocal syndrome. Whether or not hormone receptors are present in the human vocal fold has been discussed controversially. In a recently published study we investigated the expression of estrogen- $\alpha$ receptors (ER- $\alpha$ ), progesterone receptors (PR) and androgen receptors (AR) in human vocal folds by immunohistochemistry in cadaver specimens taken approximately 4-8 h postmortem [14]. We could not detect expression of the analyzed hormone receptors. Our results contradicted data presented by other authors on the expression of sex hormone receptors in laryngeal tissue [15, 16]. Although sex hormone receptors could be found in other human tissues even several hours postmortem, down-regulation or degradation of sex hormone receptors cannot be excluded. Since the local impact of estrogen and progesterone on vocal fold nodules, polyps and edema is a controversial issue, the presence of sex hormone receptors in benign vocal fold alterations needs to be clarified.

It was the goal of our study to investigate the expression of ER- $\alpha, E R-\beta, P R$ and AR in acquired benign vocal fold lesions.

\section{Material and Methods}

Intraoperatively taken specimens of 14 patients were investigated using immunohistochemistry in order to objectify ER- $\alpha$, ER- $\beta, P R$ and AR receptors. The clinical and histopathological evaluation revealed Reinke's edema in 10 patients, vocal fold polyps in 3 patients and vocal fold nodules in 1 patient. With the exception of 1 male patient (diagnosed with Reinke's edema), all patients were female. The age range was $34-78$ years, with an average of 58.2 years. All surgeries were indicated because of severe voice problems, limited verbal communication ability and lack of conservative therapy response.

All tissue samples were embedded immediately after phonosurgery in formalin and later in paraffin. The samples were first analyzed histopathologically. The remaining tissue samples underwent the standard immunohistochemical staining procedure for ER- $\alpha, P R$ and AR as described recently [14]: $4 \mu \mathrm{m}$-thick sections were cut and placed in a $65^{\circ} \mathrm{C}$ stove for $15 \mathrm{~min}$ followed by deparaffinization in xylene and rehydration in graded ethanol (ranging from 100 to $70 \%$ ). Endogenous peroxidase was blocked by $10 \mathrm{~min}$ incubation in $3 \%$ hydrogen peroxide in methanol. Antigen retrieval was carried out by microwaving the sections for 5 min at $500 \mathrm{~W}$ and $2 \times 5$ min $350 \mathrm{~W}$ successively in Citra Plus solution (BioGenex, San Ramon, Calif., USA). The biotin blocking system (Dako, Carpinteria, Calif., USA) was applied according to the instructions to reduce nonspecific staining. The following antibodies were used: (1) ER- $\alpha$ antibody - mouse monoclonal 1D5 (Dako), dilution 1:100, incubation overnight; (2) ER- $\beta$, Genetex $14 \mathrm{C} 8$, dilution $1: 200,1 \mathrm{~h}$ at room temperature; (3) PR antibody - mouse monoclonal AB-9 (Clone 1A6, NeoMarkers), readyto-use solution, and Dako M3569 or Dako clone 636, both antibodies diluted 1:1,600, incubation overnight; (4) AR antibody mouse monoclonal (BioGenex), dilution 1:50, or Dako AR441 diluted 1:100, applied at room temperature for $30 \mathrm{~min}$.

All primary antibodies were diluted with background-reducing components (Dako). The secondary antibody staining procedure for all slides was performed with ChemMate Detection Kit (Dako) that includes diaminobenzidine as a chromogen. The final staining was stopped by washing in phosphate-buffered saline. Gill's hematoxylin (Merck, Darmstadt, Germany) was used for counterstaining. Finally, sections were dehydrated and subsequently immersed in N-butyl acetate and mounted.

Human breast carcinoma tissue served as a positive control for ER- $\alpha$, ER- $\beta$ and PR, and human prostate tissue for AR. Negative controls were made by replacing the primary antibody with mouse IgG1 immunoglobulin in similar concentrations. 
The presence of brown peroxidase staining in the nucleus only was interpreted as specific positive immunoreactivity. Any cytoplasmic staining was regarded as an unspecific reaction.

\section{Data Collection and Analysis}

The slides were evaluated for specific staining patterns by members of the research team (B.S., E.C., A.K., M.v.T.) and checked for quality assurance by 2 qualified pathologists (M.R., R.H.), who were not informed about the goal of the study. All samples were blinded and judged independently by the investigators. The scoring system included a three-point scale with: $0=$ no staining, 1 = nuclear staining only, and $2=$ cytoplasmic staining (with possible staining of the nucleus).

The pathologists M.R. and R.H. are not only very experienced in identification of and interpreting data on the expression of sex hormone receptors in human tissues, but are also responsible for internal and external quality control of techniques and methods related to these data within the University Hospital of Vienna.

\section{Results}

Hormone receptors ER- $\alpha$, ER- $\beta, P R$ and also AR are expected to be nuclear. In all specimens no specific nuclear immunostaining was detectable. In several specimens unspecific brown staining was seen not only in the nuclei of epithelial and subepithelial cells, but also in cytoplasm. Expecting nuclear staining, these results had to be interpreted as unspecific reactions. Any brown cytoplasm reaction may also lead to a brown nuclear staining, thus giving the misleading impression of a specific staining. Even after modification of the staining protocols no specific nuclear staining could be obtained. While the positive controls revealed typical immunoreactivity, no nuclear staining could be seen in vocal fold specimens.

\section{Discussion}

In the current study, sex hormone receptors (ER- $\alpha$, ER- $\beta$, PR and AR) in acquired benign vocal fold lesions (Reinke's edema, vocal fold polyps, and vocal fold nodules) were examined using immunohistochemistry. Although the location of ER- $\alpha, E R-\beta, P R$ and AR is expected to be in the nucleus, we could not find any specific nuclear staining in the analyzed specimens, although we varied our staining protocols several times and obtained good results with the positive and negative controls. Thus, we have to conclude that nuclear sex hormone receptors in the larynx are not detectable by immunohistochemistry. These data are in accordance with the immunohistochemical results we gathered in vocal fold biopsies taken postmortem [14]. Voelter et al. [17] have very recently reported on the investigation of hormone receptors in human vocal fold specimens. In accordance with our data on sex hormone receptors in cadaver specimens, the authors also found no positive immunohistochemical staining in laryngeal tissue after death. In contrast to the results demonstrated in our study, Voelter et al. [17] found positive hormone receptor staining in 6 of 25 cases with vocal fold edema and in 1 of 19 patients with vocal fold polyps. Additionally, they reported on hormone receptor positivity in 3 of 4 cases with chronic laryngitis and 3 of 6 cases with leukoplakia. Considering all specimens tested Voelter et al. [17] found positive hormone receptor staining in 23 of 104 vocal folds of 97 patients.

In the past several studies have been published with inconsistent and controversial data concerning the presence of hormone receptors in the human larynx. We did not succeed in confirming the data on the expression of sex hormone receptors in human laryngeal tissue, as described previously by other authors $[15,16]$. As long as the location of ER- $\alpha$, PR and AR is expected to be in the nucleus and nuclear staining interpreted in immunohistochemistry as 'gold standard' in reference gynecopathological laboratories, the results obtained by Newman et al. [18] should be considered as 'false positive'.

Sex hormone receptors exert their function mainly as transcription factors binding to their specific binding sites on DNA. Recently, nonnuclear functions of hormone receptors have received great attention [19]. Thus, nongenomic, rapid responses to e.g. androgen receptor activation have been described [20-22]. We cannot exclude that the cytoplasmic staining found in benign vocal fold lesions may reveal hormone receptors located in the plasma membrane. Thus, further studies including a more detailed analysis need to be performed. Our limitation is the shortage of material.

In summary, sex hormone receptors could not be detected in the specimens tested. Thus, the direct influence of sex hormones on the development of benign vocal fold lesions seems rather unlikely. The results of this study confirm the impact of vocal fold stress and biomechanical abnormalities on their development due to voice overstraining and abuse.

\section{Acknowledgments}

We would like to express our sincere gratitude to Josefine Stani and the team of the Laboratory of Immunohistochemistry of the Clinical Institute of Pathology, Division of Gynecopathology, Senopathology and Cytology for their great support in performing this study. 


\section{References}

$\checkmark 1$ Gray S, Titze IR: Histologic investigation of hyperphonated canine vocal folds. Ann Otol Rhinol Laryngol 1988;97:381-388.

$\checkmark 2$ Titze IR: Mechanical stress in phonation. J Voice 1994;8:99-105.

-3 Jiang JJ, Shah AG, Hess MM, Verdolini K, Benzali FM, Hanson DG: Vocal fold impact stress analysis. J Voice 2001;15:4-14.

4 Swartz MA, Tschumperlin DJ, Kamm RD, Drazen JM: Mechanical stress is communicated between different cell types to elicit matrix modelling. Proc Natl Acad Sci USA 2001;98:6180-6185.

$\checkmark 5$ Gray SD, Hammond E, Hanson DF: Benign pathologic responses of the larynx. Ann Otol Rhinol Laryngol 1995;104:13-18.

-6 Dikkers FG, Nikkels PGJ: Benign lesions of the vocal folds: histopathology and phonotrauma. Ann Otol Rhinol Laryngol 1995; 104:698-703.

7 Johns M: Update on the etiology, diagnosis, and treatment of vocal fold nodules, polyps and cysts. Curr Opin Otolaryngol Head Neck Surg 2003;11:456-461.

-8 Gunter HE: Modeling mechanical stresses as a factor in the etiology of benign vocal fold lesions. J Biomech 2004;37:1119-1124.

$\checkmark 9$ Czerwonka L, Jiang JJ, Chao T: Vocal nodules and edema may be due to vibration-induced rises in capillary pressure. Laryngoscope $2008 ; 118: 748-752$.
10 Sułkowski WL, Kowalska S: Occupationa voice disorders: an analysis of diagnosis made and certificates issued in 1999-2004. Int J Occup Med Environ Health 2005; 18 : 341-349.

11 De Bodt MS, Ketelslagers K, Peeters T, Wuyts FL, Mertens F, Pattyn J, Heylen L, Peeters A, Boudewyns A, van de Heyning P: Evolution of vocal fold nodules from childhood to adolescence. J Voice 2007;21:151-156.

12 Amir O, Biron-Shental T: The impact of hormonal fluctuations on female vocal folds Curr Opin Otolaryngol Head Neck Surg 2004;12:180-184.

13 Abitbol J, Abitbol P, Abitbol B: Sex hormones and the female voice. J Voice 1999;13:424446.

14 Schneider B, Cohen E, Stani J, Kolbus A, Rudas M, Horvat R, van Trotsenburg M: Towards the expression of sex hormone receptors in the human vocal fold. J Voice 2007;21: 502-507.

15 Ferguson BJ, Hudson WR, McCarty KS: Sex steroid receptor distribution in the human larynx and laryngeal carcinoma. Arch Otolaryngol Head Neck Surg 1987;113:13111315.

16 Marsigliante S, Muscella A, Resta L, Storelli C: Human larynx expresses isoforms of the oestrogen receptor. Cancer Lett 1996;99: 191-196.
17 Voelter CH, Kleinsasser N, Joa P, Nowack I, Martinez R, Hagen R, Voelker HU: Detection of hormone receptors in the human vocal fold. Eur Arch Otorhinolaryngol 2008; 265:1239-1244.

18 Newman SR, ButlerJ, Hammond EH, Gray SD: Preliminary report on hormone receptors in the human vocal fold. J Voice 2000;14: $72-81$.

19 Boonyaratanakornkit V, Edwards DP: Receptor mechanisms mediating non-genomic actions of sex steroids (review). Semin Reprod Med 2007;25:139-153.

20 Cheng J, Watkins SC, Walke WH: Testosterone activates mitogen-activated protein $\mathrm{ki}$ nase via Src kinase and the epidermal growth factor receptor in Sertoli cells. Endocrinology 2007;148:2066-2074.

21 Fix C, Jordan C, Cano P, Walker WH: Testosterone activates mitogen-activated protein kinase and the cAMP response element binding protein transcription factor in Sertoli cells. Proc NatI Acad Sci USA 2004;10: 10919-10924.

22 Norman AW, Mizwicki M, Norman DP: Steroid-hormone rapid actions, membrane receptors and a conformational ensemble model. Nat Rev Drug Discov 2004;3:27-41. 